\title{
COMMENTARY
}

\section{Online Cancer Genomics Databases Provide Teaching Resources For Pharmacy Education}

\author{
Dedeepya Pasupuleti, MS, Tro Nguyen, BS, Rita Nahta, PhD \\ Mercer University, College of Pharmacy, Atlanta, Georgia \\ Corresponding Author: Rita Nahta, Mercer University, College of Pharmacy, 3001 Mercer University Dr., Atlanta, GA 30341. Tel: \\ 678-547-6347. Email: nahta_r@mercer.edu
}

Submitted April 6, 2021; accepted August 30, 2021; ePublished September 2021

Connecting scientific concepts with clinical applications is an important objective of pharmacy education. In particular, as the field of precision oncology expands, it is critical that pharmacy students understand how genetic information connects with cancer treatment decisions. However, to effectively teach students about pharmacogenomics and pharmacogenetics, faculty require relevant educational resources, including those that support higher-order learning. In this commentary, we demonstrate the potential utility of publicly accessible cancer genomics databases as teaching resources for pharmacogenomics and pharmacogenetics in oncology pharmacy education. Using clinical data retrieved from a genomics database, we illustrate how case studies can be developed to target core competencies, including understanding tumor genomics profiling, somatic mutations and pharmacotherapy selection, and clinical pharmacogenetics testing. Cancer genomics databases provide readily available, cost-effective, clinical data resources that support active learning related to pharmacogenomics and pharmacogenetics education in oncology pharmacy curricula.

Keywords: cancer genetics, education resources, oncology pharmacy, pharmacogenetics, pharmacogenomics

Teaching students how foundational science concepts apply to clinical practice is an important goal of pharmacy education ${ }^{1}$. The Accreditation Council for Pharmacy Education recommends helping "students make connections between scientific understandings and patient care" ${ }^{2}$. In the era of precision medicine, a critical objective for student pharmacists is understanding how to apply genetic information to guide treatment decisions. In their future professional roles, student pharmacists will be in key positions to educate clinical colleagues and patients about evidence-based pharmacotherapy recommendations driven by genetic information. Thus, the American College of Clinical Pharmacy (ACCP) recognizes the urgent need to include pharmacogenomics and pharmacogenetics education in pharmacy school curricula $^{3}$.

The AACP recently updated the core competencies for pharmacogenomics and pharmacogenetics consistent with those recommended by the National Human Genome Research Institute (NHGRI) ${ }^{4}$. These core competencies align with pharmacist entrustable professional activities (EPAs) and include identifying and interpreting pharmacogenomics data to determine impact on drug pharmacodynamics, response, and adverse reactions ${ }^{4}$. In addition, pharmacogenomics and pharmacogenetics educational outcomes established by the AACP Center for the Advancement of Pharmacy Education (CAPE) include understanding how genetic variants influence response to treatment and recognizing that evidence-based guidelines exist for pharmacogenetics testing and treatment selection ${ }^{5,6}$. Many pharmacy schools have added pharmacogenomics threads into disease-specific courses, including oncology. However, faculty cite a need for resources that support pharmacogenomics education, including those that can be used to develop higher-order learning activities ${ }^{7,8}$. In this commentary, we highlight the utility of publicly accessible cancer genomics databases as teaching resources for pharmacogenomics and pharmacogenetics education in oncology pharmacy curricula.

Targeted oncology drugs inhibit specific molecular drivers of cancer progression, including somatic mutations. Clinical trials of targeted drugs that include -omics analyses generally share datasets through publicly accessible cancer genomics databases. Datasets may include tumor genomics, such as differential gene analyses, somatic mutations, polymorphisms, gene amplifications, and chromosomal rearrangements, as well as pharmacotherapy and clinical outcomes data. One example of a cancer genomics database is the cBioPortal database (https://www.cbioportal.org/), which contains more than 300 datasets representing over 30 distinct tumor types ${ }^{9,10}$. The data available in each dataset vary and may include genomics, proteomics, tumor mutation count, gene amplification events, race, sex, age, tumor histopathology, treatment regimens and clinical outcomes. Due to the breadth of genomic and genetic data available from trials of targeted cancer therapies, cBioPortal represents a valuable resource for oncology pharmacogenomics education. Another cancer genomics database, Oncomine (https://www.oncomine.org), contains data from more than 4000 gene 
microarray experiments ${ }^{11}$. Oncomine supports differential gene expression analysis by tumor type, facilitating classroom discussions about pharmacogenomics and cancer precision medicine. Another resource, KM Plotter (https://kmplot.com), is designed to identify tumor markers associated with survival outcomes across 21 tumor types ${ }^{12}$. Data retrieved from KM Plotter can be used to support learning activities focused on understanding how genetic variants impact disease course and clinical outcomes.

To illustrate the application of a cancer genomics database in pharmacy education, we provide three examples using datasets from the $\mathrm{cBioPortal}$ database. The first two examples target CAPE core competencies to understand how genetic mutations inform pharmacotherapy selection based on an approved pharmacogenetics test. The third example targets a learning objective to understand immunotherapy and tumor genomics profiling. We present a case-based discussion with each example to illustrate how the data support active learning.

\section{Example 1: Discuss how a somatic mutation in PIK3CA guides treatment of breast cancer (Figure 1A).}

Data retrieval process: In this example, we started by entering a specific tumor type and drug name. Following the steps shown in the flowchart, we mined cBioPortal to obtain data for a single patient case from a clinical trial of alpelisib in breast cancer ${ }^{13}$. Case details, including tumor subtype, genetic mutation, treatment regimen and response, were downloaded from cBioPortal as shown in the table. Using these data, we created a case-based discussion (shown below) focused on treatment selection guided by molecular and genetic information, pharmacogenetic testing, and drug-drug interactions. In addition to this case, we downloaded data for a larger cohort of patients (shown in the graph) to introduce concepts related to clinical response and resistance.

Case: A postmenopausal female patient presents with advanced breast cancer. The patient's breast tumor biopsy is positive for estrogen receptor (ER) and negative for human epidermal growth factor receptor 2 (HER2). The patient is started on exemestane. Upon progressing on exemestane, pharmacogenetics testing of tumor tissue is performed for the PIK3CA gene. The patient is found to have an H1047R PIK3CA somatic mutation and is started on alpelisib plus fulvestrant.

Case study questions: (1) What are exemestane and alpelisib? (2) What information guided selection of these treatments? (3) Why does a positive pharmacogenetics test not guarantee response to a targeted treatment? (4) What drugdrug interactions need to be considered with alpelisib?

Case-based discussion guide: The questions should guide discussion about targeted therapies, including those targeting somatic mutations and companion pharmacogenetics testing. Discussion should also note variability in patient responses and could include discussions about intra- and inter-tumoral heterogeneity, patient-specific factors influencing response, and acquired drug resistance. Finally, drug-drug interactions and the effects of cytochrome p450 (CYP) enzymes on drug activity should be discussed.

\section{Example 2: Discuss how a somatic mutation in $B R A F$ guides treatment of melanoma (Figure 1B).}

Data retrieval process: In this example, we started by entering a specific cancer type and gene name. Similar to the process described above, we mined cBioPortal to obtain a single patient case of $B R A F$-mutant melanoma following the steps in the flowchart ${ }^{14}$. In addition to the single case, we downloaded a graph showing responses in a larger cohort of patients with $B R A F$-mutant melanoma treated with dabrafenib or vemurafenib to discuss other compounds in this class of drugs. We also introduce the concept of pharmacogenetics testing to assess a marker associated with adverse events.

Case: A 44-year old female patient with metastatic melanoma is found to have a somatic $B R A F^{\mathrm{V} 600 \mathrm{E}}$ mutation. The patient is also found to have wild-type alleles for the glucose-6-phosphate dehydrogenase (G6PD) gene. The patient is treated with dabrafenib for 30 weeks with minimal toxicity and exhibits complete response to therapy.

Case study questions: (1) What information guided the selection of dabrafenib as a treatment in this patient? (2) What is the mechanism of action of dabrafenib and vemurafenib? (3) Why is pharmacogenetics testing for the G6PD gene important when considering dabrafenib as a potential treatment?

Case-based discussion guide: The questions should guide discussion of $B R A F$ mutations, pharmacogenetics testing for somatic mutations, and mechanisms of action of kinase-targeted therapy. The question about G6PD testing should guide discussion about patient-specific factors that may influence adverse drug effects.

\section{Example 3: Discuss PD-1-directed immunotherapy and tumor genome alterations (Figure 2).}

Data retrieval process: In this example, we started by entering a drug name and mined cBioPortal to display tumor gene analysis from a translational study in patients with melanoma ${ }^{15}$. We downloaded differential gene expression data for pre-treatment tumor biopsies of responders and non-responders and a case profile to discuss concepts related to immunotherapy and tumor genomics. 
Case: An 84-year old male patient with metastatic melanoma has progressed on prior therapy with a mitogenactivated protein kinase (MAPK) inhibitor. The patient is treated with pembrolizumab as part of a trial evaluating pembrolizumab or nivolumab in patients with melanoma. Immune cell profiling and pre-treatment tumor genomics profiling are performed as part of this trial.

Case study questions: (1) What is the mechanism of action of pembrolizumab and nivolumab? (2) Why was immune cell profiling performed? (3) What do the data in the tumor genomics table show?

Case-based discussion guide: The questions should guide discussion about cancer immunotherapy, immune cell types and functions, and the role of genomics data in biomarker and drug discovery research.

These examples are provided to illustrate the potential utility of genomics databases for case study development in the area of cancer pharmacogenomics and pharmacogenetics. Although student feedback, perceptions and knowledge gains remain to be assessed, we anticipate a number of advantages to this approach. First, integrating clinical datasets into active learning may assist students in applying foundational concepts to solve practical problems ${ }^{16}$, such as the use of genetics data to create informed treatment plans. Second, faculty who lack clinical context may benefit from the ability to retrieve real-life cases from genomics databases, rather than creating hypothetical scenarios. Thus, clinical genomics databases may support pre-course preparation and potentially reduce interdisciplinary workload ${ }^{17}$. Third, students can be introduced to emerging pharmacogenomics research, which may enhance their awareness of academic research and related career opportunities ${ }^{18,19}$. Fourth, the functions we used to retrieve data were freely accessible, making these costeffective options ${ }^{19}$. Pharmacy programs with limited resources can integrate genomics databases into mentored research experiences to foster critical thinking and problem solving ${ }^{18,19}$. Finally, online databases can be used in synchronous, asynchronous, in-person, blended, and virtual learning environments, making them versatile resources.

Despite the benefits of publicly accessible cancer genomics databases, there are limitations. Due to a wide range of user interfaces, databases vary in complexity and the amount of time and skill required to retrieve and analyze data. Additionally, although there are many publicly accessible databases suitable for oncology pharmacy education, faculty must be made aware of these resources and know where to find datasets appropriate for their specific learning objectives. We found online tutorials, manuals, and published research articles helpful as we learned to use these databases.

Incorporating clinical genomics data into active learning targets a number of pharmacy student core competencies, such as identifying and interpreting pharmacogenomics test results, determining the impact of genetic variants on drug activity and adverse events, and educating others about the applications of genetic information for optimal drug therapy ${ }^{4}$. These competencies map to multiple EPA domains, including patient care provider, population health promoter, and information master. Genomics databases also address a number of CAPE outcomes, including integrating and applying foundational knowledge (domain 1), interpreting data (domain 2), and problem solving (domain $3)^{20}$. Ultimately, understanding the application of genetics to inform treatment selection, gaining awareness of evidencebased guidelines for pharmacogenetics testing, and developing a strong knowledge of precision cancer medicine are important objectives targeted by these resources. Thus, publicly accessible cancer genomics databases represent rich sources of clinical pharmacogenomics and pharmacogenetics data that can be adapted for active learning in oncology pharmacy education.

\section{ACKNOWLEDGMENTS}

We thank Dr. Milap Nahata, PharmD, for critically reviewing this commentary.

Conflicts of Interest and Disclosures: RN reports personal holdings in GlaxoSmithKline PLC., Pfizer, Bristol Myers Squibb, and Eli Lilly and Company.

\section{REFERENCES}

1. Lerchenfeldt SH, L.M. Pharm.D.s in the Midst of M.D.s and Ph.D.s: the Importance of Pharmacists in Medical Education. Medical Science Educator. 2018;28:259-261.

2. Guidance for the Accreditation Standards and Key Elements for the Professional Program in Pharmacy Leading to the Doctor of Pharmacy Degree. Accreditation Council for Pharmacy Education. 2016.

3. Hicks JK, Aquilante CL, Dunnenberger HM, et al. Precision Pharmacotherapy: Integrating Pharmacogenomics into Clinical Pharmacy Practice. J Am Coll Clin Pharm. Jun 2019;2(3):303-313. doi:10.1002/jac5.1118

4. Gammal RS, Lee YM, Petry NJ, et al. Pharmacists Leading the Way to Precision Medicine: Updates to the Core Pharmacist Competencies in Genomics. Am J Pharm Educ. Jul 22 2021:8634. doi:10.5688/ajpe8634

5. Roederer MW, Kuo GM, Kisor DF, et al. Pharmacogenomics competencies in pharmacy practice: A blueprint for change. J Am Pharm Assoc (2003). Jan - Feb 2017;57(1):120-125. doi:10.1016/j.japh.2016.08.014

6. Farrell CL KD, Smith HE, et al. The DNA of Pharmacy Education: CAPE Outcomes and Pharmacogenomics. Accessed July 6, 2021, http://www.aacp.org/resources/education/cape/Pages/default.aspx 
7. Crabtree B, Bootman JL, Boyle CJ, Chase P, Piascik P, Maine LL. Aligning the AACP Strategic Engagement Agenda with Key Federal Priorities in Health: Report of the 2016-17 Argus Commission. Am J Pharm Educ. Oct 2017;81(8):S15. doi:10.5688/ajpeS15

8. Weitzel KW, Aquilante CL, Johnson S, Kisor DF, Empey PE. Educational strategies to enable expansion of pharmacogenomics-based care. Am J Health Syst Pharm. Dec 1 2016;73(23):1986-1998. doi:10.2146/ajhp160104

9. Cerami E, Gao J, Dogrusoz U, et al. The cBio cancer genomics portal: an open platform for exploring multidimensional cancer genomics data. Cancer Discov. May 2012;2(5):401-4. doi:10.1158/2159-8290.CD-12-0095

10. Gao J, Aksoy BA, Dogrusoz U, et al. Integrative analysis of complex cancer genomics and clinical profiles using the cBioPortal. Sci Signal. Apr 2 2013;6(269):pl1. doi:10.1126/scisignal.2004088

11. Rhodes DR, Yu J, Shanker K, et al. ONCOMINE: a cancer microarray database and integrated data-mining platform. Neoplasia. Jan-Feb 2004;6(1):1-6. doi:10.1016/s1476-5586(04)80047-2

12. Nagy A, Munkacsy G, Gyorffy B. Pancancer survival analysis of cancer hallmark genes. Sci Rep. Mar 15 2021;11(1):6047. doi:10.1038/s41598-021-84787-5

13. Razavi P, Dickler MN, Shah PD, et al. Alterations in PTEN and ESR1 promote clinical resistance to alpelisib plus aromatase inhibitors. Nat Cancer. Apr 2020;1(4):382-393. doi:10.1038/s43018-020-0047-1

14. Van Allen EM, Wagle N, Sucker A, et al. The genetic landscape of clinical resistance to RAF inhibition in metastatic melanoma. Cancer Discov. Jan 2014;4(1):94-109. doi:10.1158/2159-8290.CD-13-0617

15. Hugo W, Zaretsky JM, Sun L, et al. Genomic and Transcriptomic Features of Response to Anti-PD-1 Therapy in Metastatic Melanoma. Cell. Mar 24 2016;165(1):35-44. doi:10.1016/j.cell.2016.02.065

16. Greene JM, Fuller KA, Persky AM. Practical Tips for Integrating Clinical Relevance into Foundational Science Courses. Am J Pharm Educ. Jun 2018;82(5):6603. doi:10.5688/ajpe6603

17. Islam MA, Talukder RM, Taheri R, Blanchard N. Integration of Basic and Clinical Science Courses in US PharmD Programs. Am J Pharm Educ. Dec 25 2016;80(10):166. doi:10.5688/ajpe8010166

18. Deal EN, Stranges PM, Maxwell WD, et al. The Importance of Research and Scholarly Activity in Pharmacy Training. Pharmacotherapy. Dec 2016;36(12):e200-e205. doi:10.1002/phar.1864

19. Perez A, Rabionet S, Bleidt B. Teaching Research Skills to Student Pharmacists in One Semester: An Applied Research Elective. Am J Pharm Educ. Feb 25 2017;81(1):16. doi:10.5688/ajpe81116

20. Medina MS, Plaza CM, Stowe CD, et al. Center for the Advancement of Pharmacy Education 2013 educational outcomes. Am J Pharm Educ. Oct 14 2013;77(8):162. doi:10.5688/ajpe 778162 
Figure 1. Examples of Pharmacogenetics Case Studies from the cBioPortal Cancer Genomics Database

\section{A. Case of complete response to alpelisib in PIK3CA-mutant breast cancer}

\begin{tabular}{|c|}
\hline https://www.cbioportal.org/ \\
\hline 1 \\
\hline Select "Breast Cancer" \\
\hline 7 \\
\hline Search for "alpelisib" \\
\hline 2 \\
\hline $\begin{array}{l}\text { Select all listed studies } \\
\text { matching filter }\end{array}$ \\
\hline 2 \\
\hline $\begin{array}{c}\text { Click "Explore Selected } \\
\text { Studies" }\end{array}$ \\
\hline 1 \\
\hline $\begin{array}{l}\text { Select "PIK3CA" and "145" } \\
\text { for \# under "Mutated Genes" }\end{array}$ \\
\hline 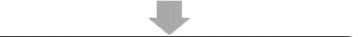 \\
\hline Click "Select Samples" \\
\hline 1 \\
\hline $\begin{array}{l}\text { Under "Charts", click } \\
\text { "treatment best response" }\end{array}$ \\
\hline 1 \\
\hline $\begin{array}{l}\text { In the "trcatment best } \\
\text { response" chart, click "show } \\
\text { table" }\end{array}$ \\
\hline 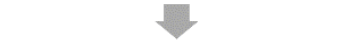 \\
\hline $\begin{array}{l}\text { Select the box next to } \\
\text { "complete response" }\end{array}$ \\
\hline 1 \\
\hline $\begin{array}{c}\text { Under "clinical data", click on } \\
\text { paticnt ID }\end{array}$ \\
\hline$y$ \\
\hline $\begin{array}{l}\text { The tab "clinical data" } \\
\text { contains the case profile }\end{array}$ \\
\hline
\end{tabular}

\begin{tabular}{|ll|}
\hline \multicolumn{1}{|c|}{ Pattribute } & \multicolumn{1}{c|}{ Value } \\
\hline Treatment Arm & D \\
Treatment Best Response & Complete Response \\
Alpelisib Dose (mg) & 350 \\
Alpelisib Scheduling & Intermittent (5/2) \\
Endocrine Therapy & Exemestane \\
Measurable Disease & Yes \\
Weeks on Study & 150 \\
Best RECIST Response & -100 \\
Breast Cancer Subtype & IIR+/HER2- \\
Clinical Benefit & Yes \\
Number of Samples Per Patient & 5 \\
Off Study Reason & POD \\
Paticnt Display Namc & P042 \\
PIK3CA Pre Treatment Tumor & MUT \\
PIK3CA Pre Treatment Tumor Mutation & H1047R \\
Sex & Female \\
\hline
\end{tabular}

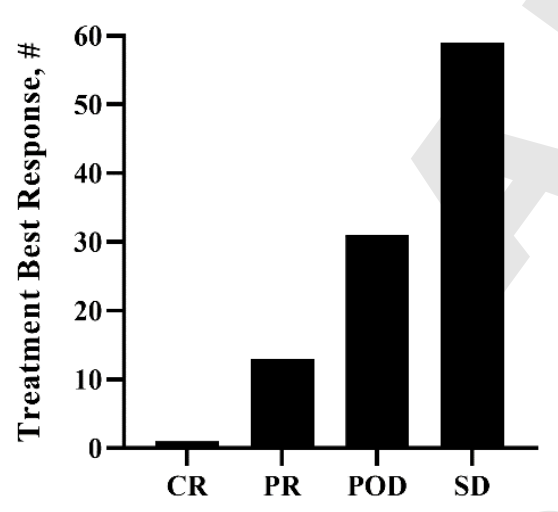

CR PR POD SD

\section{B. Case of complete response to dabrafenib in $B R A F$-mutant melanoma}

\begin{tabular}{|c|c|c|}
\hline \multirow{2}{*}{ https://www.cbioportal.org/ } & \multicolumn{2}{|c|}{ Patient ID: Pat_21 } \\
\hline & Attribute & Value \\
\hline$\gamma$ & Diagnosis $\Lambda$ ge & 44 \\
\hline Select "Skin Cancer" & duration of therapy weeks & 30 \\
\hline$\overline{1}$ & Early Resistance & No \\
\hline Search for "bral" & medication & Dabrafenib \\
\hline 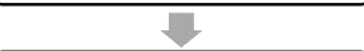 & Sex & Female \\
\hline $\begin{array}{l}\text { Select all listed studies } \\
\text { matching filter }\end{array}$ & $\begin{array}{l}\text { Treatment best response } \\
\text { Mutation Count }\end{array}$ & $\begin{array}{l}\mathrm{CR} \\
31\end{array}$ \\
\hline 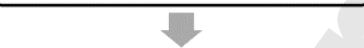 & Cancer Typc & Mclanoma \\
\hline $\begin{array}{l}\text { Click "Explore Selected } \\
\text { Studies" }\end{array}$ & $\begin{array}{l}\text { Cancer Type Detailed } \\
\text { Matched Normal Tissue }\end{array}$ & $\begin{array}{l}\text { Melanoma } \\
\text { Paraffin Embedded Tissue }\end{array}$ \\
\hline 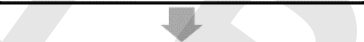 & mean target coverage normal & 104 \\
\hline $\begin{array}{l}\text { Select "BRAF" and " } 67 \text { " for \# } \\
\text { under "Mutated Genes" }\end{array}$ & $\begin{array}{l}\text { mean target coverage tumor } \\
\text { Oncotree Code }\end{array}$ & $\begin{array}{l}82 \\
\text { MEL }\end{array}$ \\
\hline 5 & Somatic Status & Matched \\
\hline
\end{tabular}

Click "Select Samples"

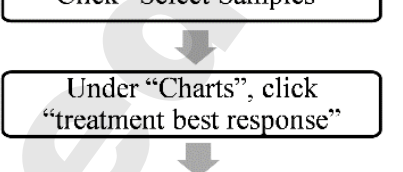

In the "treatment best

response" chart, click "show table"

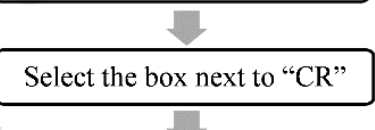
$\checkmark$

Under "clinical data", click on patient ID

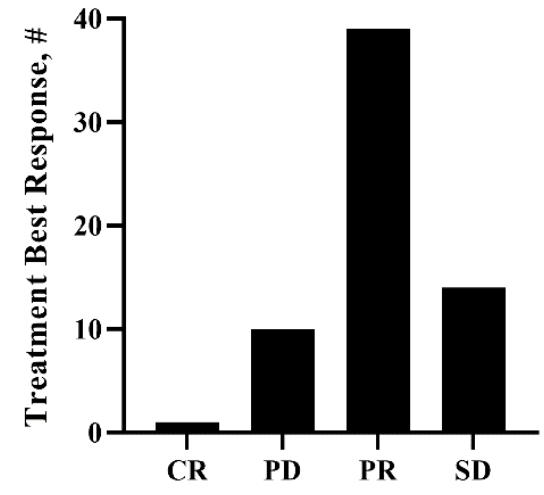

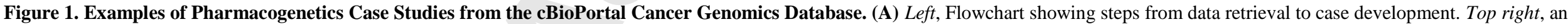

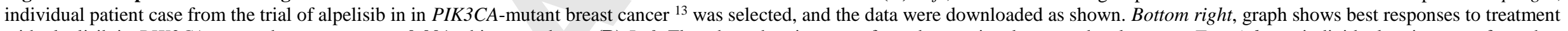

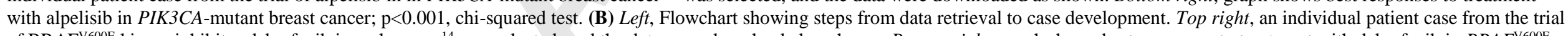

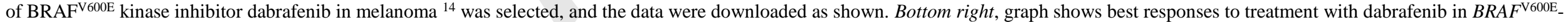
mutant melanoma; $\mathrm{p}<0.001$, chi-squared test. $\mathrm{CR}$, complete response; PR, partial response; PD, progressive disease; POD, progression of disease; $\mathrm{SD}$, stable disease. 
Figure 2. Example of Pharmacotherapy Gene Analysis from the cBioPortal Cancer Genomics Database

\section{Flowchart}

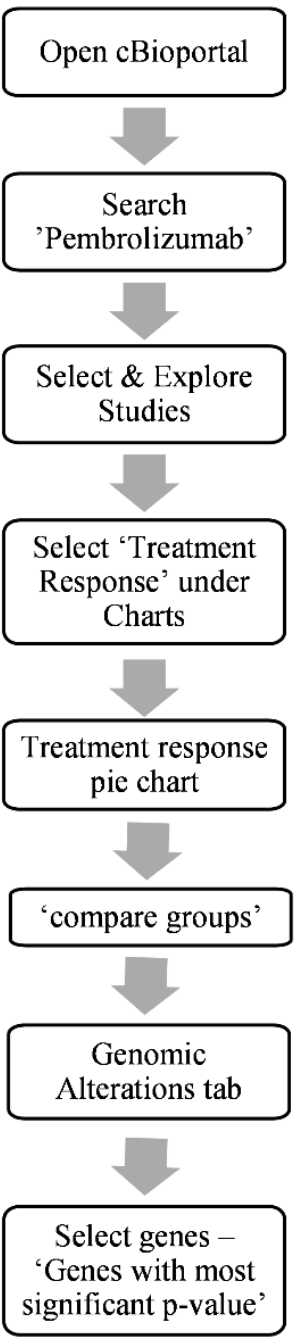

Gene alterations in patients with metastatic melanoma treated with pembrolizumab plus nivolumab

\begin{tabular}{|c|c|c|c|c|c|}
\hline Gene & Cytoband & (A) Non-responder & (B) Responder & p-Value & Enriched in \\
\hline USP6 & $17 \mathrm{p} 13.2$ & $0(0.00 \%)$ & $10(47.62 \%)$ & $7.46 \mathrm{E}-04$ & (B) Responder \\
DST & $6 \mathrm{p} 12.1$ & $0(0.00 \%)$ & $9(42.86 \%)$ & $1.80 \mathrm{E}-03$ & (B) Responder \\
MDN1 & $6 \mathrm{q} 15$ & $0(0.00 \%)$ & $9(42.86 \%)$ & $1.80 \mathrm{E}-03$ & (B) Responder \\
PRAMEF4 & $1 \mathrm{p} 36.21$ & $0(0.00 \%)$ & $9(42.86 \%)$ & $1.80 \mathrm{E}-03$ & (B) Responder \\
UTRN & $6 \mathrm{q} 24.2$ & $0(0.00 \%)$ & $8(38.10 \%)$ & $4.16 \mathrm{E}-03$ & (B) Responder \\
DSP & $6 \mathrm{p} 24.3$ & $1(5.88 \%)$ & $10(47.62 \%)$ & $5.28 \mathrm{E}-03$ & (B) Responder \\
LRP4 & $11 \mathrm{p} 11.2$ & $7(41.18 \%)$ & $1(4.76 \%)$ & $8.85 \mathrm{E}-03$ & (A) Non-responder \\
MAP3K21 & $1 \mathrm{q} 42.2$ & $7(41.18 \%)$ & $1(4.76 \%)$ & $8.85 \mathrm{E}-03$ & (A) Non-responder \\
NAV1 & $1 \mathrm{q} 32.1$ & $7(41.18 \%)$ & $1(4.76 \%)$ & $8.85 \mathrm{E}-03$ & (A) Non-responder \\
CLUH & $17 \mathrm{p} 13.3$ & $0(0.00 \%)$ & $7(33.33 \%)$ & $9.21 \mathrm{E}-03$ & (B) Responder \\
\hline
\end{tabular}

\section{Case profile}

\begin{tabular}{|ll|}
\hline \multicolumn{2}{|c|}{ Pattribute } \\
\hline \multicolumn{1}{|c|}{ Attient ID: PT29 } \\
\hline Overall Survival (Months) & \multicolumn{1}{c|}{ Value } \\
Overall Survival Status & 1:DECEASED \\
Durable Clinical Benefit & PD \\
M Stage & M1c \\
Previous MAPKi & Yes \\
Sex & Male \\
Number of Samples Per Patient & 1 \\
Age at Diagnosis & 84 \\
Treatment & Pembrolizumab \\
\hline
\end{tabular}

\begin{tabular}{|ll|}
\hline \multicolumn{1}{|c|}{ Samples } \\
\hline \multicolumn{1}{|c|}{ Attribute } & \multicolumn{1}{c|}{ Pt29 } \\
\hline Mutation Load & 313 \\
Neo-antigen Load & 437 \\
ESTIMATE Immune Score & 1724.62 \\
CBSET T cells CD8 & 0.24 \\
CBSET Macrophages M0 & 0 \\
CBSET Macrophages M1 & 0.13 \\
CBSET Macrophages M2 & 0.29 \\
CBSET T cells CD4 memory activated & 0.02 \\
CBSET Dendritic cells activated & 0 \\
HLA-A & A0201/A0201 \\
HLA-B & B4402/B5701 \\
HLA-C & C0501/C0602 \\
Cancer Type & Melanoma \\
Treatment Response & Non-responder \\
Biopsy Time & Pre-treatment \\
CBSET B cells memory & 0 \\
CBSET B cells naive & 0.06 \\
CBSET NK cells activated & 0.03 \\
CBSET NK cells resting & 0 \\
CBSET T cells CD4 memory resting & 0.09 \\
CBSET T cells CD4 naive & 0 \\
CBSET T cells regulatory (Tregs) & 0 \\
\hline
\end{tabular}


Figure 2. Example of Pharmacotherapy Gene Analysis Case Study from the cBioPortal Cancer Genomics Database. (A) Left, Flowchart showing steps for retrieving pharmacogenomics table. Top right, table of differential gene analysis in pre-treatment tumor tissues from responders and non-responders to pembrolizumab and nivolumab ${ }^{15}$. Bottom right, an individual patient case was selected, and the clinical and immune cell profiling data were downloaded as shown. 